\title{
Structure and Properties of Polynorbornene Derivatives: Poly(norbornene dicarboxylic acid dialkyl ester)s and Poly(norbornene dimethyl dicarboxylate)s
}

\author{
Boo-Gyo Shin, Tai-Yon Cho, and Do Y. Yoon* \\ Department of Chemistry, Seoul National University, Seoul 151-747, Korea \\ Binyuan Liu \\ Institute of Polymer Science \& Engineering, Hebei University of Technology, Tianjin, 300130, China
}

Received November 23, 2006; Revised December 14, 2006

\begin{abstract}
Poly(norbornene dimethyl dicarboxylate)s, (PNDMD)s, were prepared by addition polymerization with palladium(II) catalyst from pure exo-monomers, and their structure and properties were compared with those of poly(norbornene dicarboxylic acid dialkyl ester)s, (PNDADA)s. Both polymer series exhibited good solubility in general organic solvents and excellent thermal stability up to $330{ }^{\circ} \mathrm{C}$. Wide-angle X-ray scattering (WAXS) study indicated the presence of nano-scale layer-like order in amorphous PNDADAs, while PNDMDs showed random amorphous structure. The glass transition temperatures and dielectric constants of solid polymers were found to decrease as the alkyl side-chain length increases for both polymer series. However, PNDMDs showed lower glass transition temperatures and higher dielectric constants, as compared with those of PNDADAs containing the same alkyl substituents. This difference, caused by the higher side-group mobility of PNDMDs, may be closely related to the nano-scale order in amorphous PNDADAs and its absence in PNDMDs.
\end{abstract}

Keywords: polynorbornene, polynorbornene derivatives, thermal stability, glass transition, dielectric constant, optical properties.

\section{Introduction}

Vinyl-polynorbornene has good physical characteristics desired for the application in information technology devices, such as excellent optical transparency, high thermal stability up to $400{ }^{\circ} \mathrm{C}$, high glass transition temperature $\left(T_{g}\right)$ above $300^{\circ} \mathrm{C}$, low moisture absorption, and a low dielectric constant (ca. 2.5). ${ }^{1,2}$ However, its poor solubility in common solvents, mechanical brittleness, and low adhesion property present serious drawbacks towards potential applications. ${ }^{1,2}$

Vinyl-polynorbornene derivatives with suitable side-chains could improve the solubility and adhesion properties. For example, Goodall et al. synthesized functionalized polynorbornene with alkyl groups, which improved the mechanical properties of the polymer and resulted in higher elongationto-break values. ${ }^{3}$ Moreover, functionalized polynorbornene with triethoxysilyl group improved the adhesion characteristics by hydrolysis of ethoxy group followed by condensation reaction of hydroxy group to the substrate.,

*Corresponding Author. E-mail: dyyoon@snu.ac.kr
However, polymerization of functionalized norbornene is more difficult than that of norbornene. Polymerization rate decreases drastically as polar side-groups are introduced, since the coordination of functional group with the catalyst decreases the catalyst activity. Moreover, the polymerization of endo-functionalized norbornenes is more difficult than that of exo-functionalized norbornenes, since the endo-functionalized norbornene could coordinate more strongly with the catalyst, as compared with the exo counterpart. ${ }^{6-8}$

In this paper, we report the preparation of exo-norbornene dimethyl dicarboxylates with various alkyl substituents and addition polymerization of the exo-monomers by Pd catalyst. The structure and properties of poly(norbornene dimethyl dicarboxylate)s, (PNDMD)s, were characterized and compared with those of poly(norbornene dicarboxylic acid dialkyl ester)s, (PNDADA)s, whose synthesis was reported previously. ${ }^{9}$ In particular, the local amorphous structure of PNDMDs and PNDADAs was investigated by wide-angle X-ray scattering (WAXS) experiments. And the differences in the glass transition characteristics and the dielectric constants are discussed on the basis of this structural model. 


\section{Experimental}

Thermal gravimetric analysis (TGA) experiments were performed using TGA 2050 (TA instruments). Polymer samples were heated at a rate of $10 \mathrm{~K} / \mathrm{min}$ under nitrogen. WAXS experiments were performed using M18XHF-SRA (MAC Science Co.). Data were obtained with $\mathrm{Cu} K_{\alpha}$ source with $\lambda=1.54 \AA$ in the range of $2 \theta_{B}=3.5 \sim 30^{\circ}$. Dynamic mechanical analysis (DMA) experiments were performed using DMA 2980 (TA instruments). Samples for DMA measurements were prepared by dissolving in THF, coating on glass fibers, and drying for $12 \mathrm{hrs}$ under vacuum. The samples on glass fibers were heated at $2 \mathrm{~K} / \mathrm{min}$, and data were obtained at $1 \mathrm{~Hz}$ frequency and $30 \mu \mathrm{m}$ amplitude in dual-cantilever mode under nitrogen. Dielectric constant was measured by metal-insulator-metal (MIM) parallel plate capacitance method. For the preparation of MIM samples, polymer solution was spin-coated on a heavily doped silicon wafer, and upon removal of solvents by vacuum drying, the top Al electrode was deposited by thermal evaporation on the polymer film. Capacitance was measured at $1 \mathrm{MHz}$ by LCR meter (Agilent 4294A). Refractive index was measured at $632.8 \mathrm{~nm}$ by thin-film analyzer, Filmetrics F20 (Filmetrics, Inc.).

\section{Results and Discussion}

Synthesis. A new series of poly(norbornene dimethyl dicarboxylate)s (poly-A, B, C, D, E) were synthesized as shown in Figure 1. To obtain pure exo-monomers, 5-nor-<smiles>O=C1OC(=O)C2CCC1C2</smiles>

$185^{\circ} \mathrm{C}$

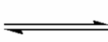

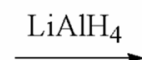

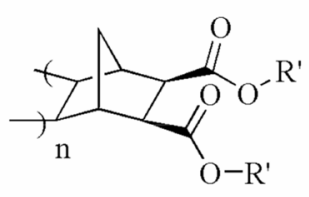

R' : Me, Et, Pr, Bu, Pen, Hex, 2-ethyl-hexyl Poly-1,2,3,4,5,6,7 Series
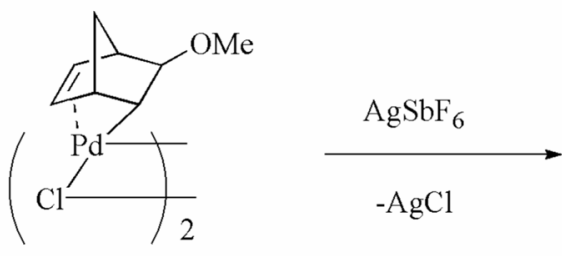<smiles>[R]C(=O)OCC1C2C=CC(C2)C1CO</smiles><smiles>[R]C(=O)OCC1C2C=CC(C2)C1COC([R])=O</smiles>

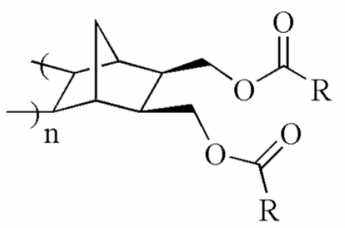

$\mathrm{R}: \mathrm{Me}, \mathrm{Et}, \mathrm{Pr}, \mathrm{Bu}, \mathrm{Pen}$ Poly-A,B,C,D,E Series

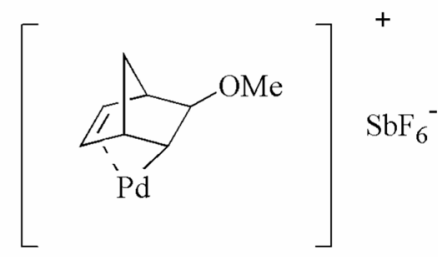

Catalyst I

Figure 1. Reaction scheme for preparing monomers, polymers, and catalyst. 
bornene-endo-2,3-dicarboxylic anhydride was rearranged at $185^{\circ} \mathrm{C}$, and 5-norbornene-exo-2,3-dicarboxylic anhydride was isolated by recrystallization in more than $98 \%$ exopurity. ${ }^{10}$ 5-Norbornene-exo-2,3-dicarboxylic anhydride was reduced to 2,3-exo-bis(hydroxymethyl)norbornene by $\mathrm{LiAlH}_{4}$. Various norbornene dimethyl dicarboxylates with various alkyl substituents could be prepared by esterification of 2,3bis(hydroxymethyl)norbornene and acyl halide with diverse alkyl chain lengths. Exo-monomers were further purified by vacuum distillation except for monomer-D, E for which the boiling points are too high for vacuum distillation. Addition polymerizations of exo-monomers with catalyst $\mathbf{I}$ in chlorobenzene yielded poly(norbornene dimethyl dicarboxylate)s. Reaction solution became viscous after several hours. Catalyst was removed by reducing with $\mathrm{H}_{2}$ gas to $\mathrm{Pd}(0)$ aggregates. ${ }^{11}$ Full details of the monomer synthesis and the preparation of the polymer samples employed in this study will be presented separately. ${ }^{12}$

Previously, the synthesis of poly(norbornene dicarboxylic acid dialkyl ester)s (poly-1, 2, 3, 4, 5, 6) were reported in detail, together with the variation of their thermal stabilities and glass transition temperatures with the different alkyl substituents. ${ }^{9}$

The molecular weights as estimated from the gel perme- ation chromatography (GPC) with polystyrene standards are listed in Table I, together with the measured polymerization yields.

Solubility Characteristics. Solubility was tested at room temperature for $10 \mathrm{wt} \%$ concentrations. Both PNDMDs (poly-A, B, C, D, E) and PNDADAs (poly-1, 2, 3, 4, 5, 6, 7) showed good solubility in a number of common solvents such as toluene, ethyl acetate, THF, dichloromethane, chloroform and acetone, as shown in Table II. They are insoluble in hexane and methanol, however. For comparison, amorphous polynorbornene is soluble only in limited solvents such as unsaturated halogenated hydrocarbons. ${ }^{7}$

Thermal Decomposition Characteristics. The TGA thermograms of poly-A, B, C, D, E are shown in Figure 2, together with the corresponding results for polynorbornene. Although the thermal stability is lower as compared with polynorbornene, PNDMDs still exhibited good thermal stability at high temperatures up to $330^{\circ} \mathrm{C}$, and their thermograms showed a broad sigmoidal weight loss profile with the thermal decomposition occurring from 330 to $450{ }^{\circ} \mathrm{C}$. There was little difference among PNDMD polymers with different alkyl substituents, very similar to the previous finding for poly(alkyl-norbornene)s. ${ }^{13}$ In comparison, PNDADAs (poly-2, 3, 4, 5, 6) exhibited thermograms with

Table I. Molecular Weight, Polymerization Yield, Glass Transition Temperature $\left(T_{g}\right)$, Dielectric Constant $(\varepsilon)$ and Refractive Index (n) of Polymers

\begin{tabular}{|c|c|c|c|c|c|c|}
\hline & $M_{w}{ }^{a}$ & $M_{n}{ }^{a}$ & Yield (\%) & $T_{g}\left({ }^{\circ} \mathrm{C}\right)$ & $\varepsilon^{b}$ & $n^{c}$ \\
\hline Poly-A & 66,000 & 50,000 & 81 & 331 & 3.5 & 1.52 \\
\hline Poly-B & 72,000 & 60,000 & 88 & 324 & 3.5 & 1.52 \\
\hline Poly-C & 205,000 & 133,000 & 68 & 318 & 3.4 & 1.52 \\
\hline Poly-D & 223,000 & 121,000 & 92 & 276 & 3.3 & 1.51 \\
\hline Poly-E & 78,000 & 31,000 & 65 & - & 3.0 & 1.50 \\
\hline Poly-1 & 116,000 & 82,000 & 95 & - & 3.3 & 1.51 \\
\hline Poly-2 & 48,000 & 32,000 & 86 & - & 3.1 & 1.50 \\
\hline Poly-3 & 106,000 & 84,000 & 67 & 322 & 2.9 & 1.50 \\
\hline Poly-4 & 62,000 & 53,000 & 69 & 305 & 2.9 & 1.50 \\
\hline Poly-5 & 78,000 & 51,000 & 88 & 230 & 2.6 & 1.50 \\
\hline Poly-6 & 81,000 & 62,000 & 69 & 203 & 2.6 & 1.46 \\
\hline Poly-7 & 29,000 & 12,000 & 80 & - & - & \\
\hline
\end{tabular}

${ }^{a}$ Polystyrene standard-based values from GPC. ${ }^{b}$ Measured at $1 \mathrm{MHz} .{ }^{c}$ Measured at $632.8 \mathrm{~nm}$.

Table II. Solubility Test for 10 wt\% Solution at Room Temperature

\begin{tabular}{lcccc}
\hline & Hexane & Toluene & $\begin{array}{c}\text { Ethyl acetate, THF, } \\
\mathrm{CH}_{2} \mathrm{Cl}_{2}, \mathrm{CHCl}_{3}\end{array}$ & Acetone \\
\hline Poly-A & $\times$ & $\times$ & $\bigcirc$ & $\bigcirc$ \\
Poly-B, C, D, E & $\times$ & $\bigcirc$ & $\bigcirc$ & $\bigcirc$ \\
Poly-1 & $\times$ & $\times$ & $\bigcirc$ & $\bigcirc$ \\
Poly-2 & $\times$ & $\triangle$ & $\bigcirc$ & $\bigcirc$ \\
Poly-3, 4, 5 & $\times$ & $\bigcirc$ & $\bigcirc$ & $\bigcirc$ \\
Poly-6 & $\times$ & $\bigcirc$ & $\bigcirc$ & $\times$ \\
Poly-7 & $\bigcirc$ & $\bigcirc$ & $\bigcirc$ & $\times$ \\
\hline
\end{tabular}

$\times$ : insoluble, $\triangle$ : partly soluble, $\bigcirc$ : soluble. 


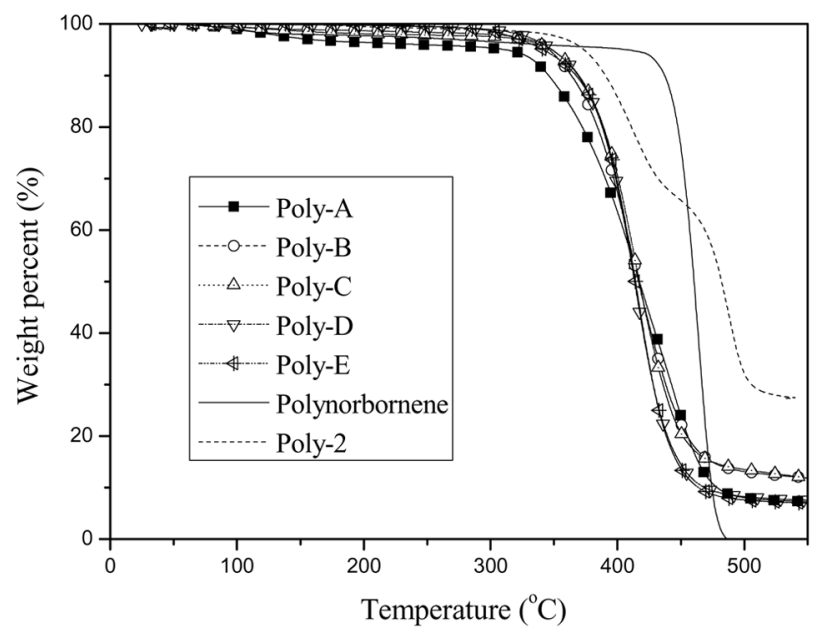

Figure 2. TGA thermograms of poly-A, B, C, D, E, obtained at $10 \mathrm{~K} / \mathrm{min}$ heating rate under nitrogen environment, in comparison with the results of polynorbornene and poly-2.

two discrete thermal decomposition steps, as exemplified by poly-2 in Figure 2, which was interpreted as arising from the initial side-chain decomposition by $\beta$-hydrogen elimination followed by main-chain decomposition.

Wide Angle X-Ray Scattering (WAXS) Results. As is well known, amorphous polynorbornene shows two broad amorphous halo peaks at $2 \theta_{B}=10$ and $18^{\circ}$, respectively. The $2 \theta_{B}$ peak at $10^{\circ}$ is interpreted to arise from the interchain spacing of $8.6 \AA$, and the peak at $18^{\circ}$ is due to the typical spacing of $4.8 \AA$ between the neighboring $\mathrm{CH}_{2}$ groups. ${ }^{14,15}$ For poly(alkyl-norbornene) (alkyl=methyl, butyl, and hexyl), the distance between main chains increases with the increasing alkyl length due to the steric repulsion between the attached alkyl groups, resulting in the low angle peaks shifting to lower $2 \theta_{B}$ positions with decreasing peak intensity. ${ }^{16}$

In the case of poly-A, B, C, D, E series, our WAXS spectra are similar to those of polynorbornene and poly(alkyl norbornene), with the Bragg $d$-spacing of the low angle peak increasing with the alkyl length (see Figure 3 and Table III). In comparison, the WAXS patterns for PNDADAs (poly-1, $2,3,4)$ are more complicated. There are 2 or 3 amorphous halo peaks as shown in Figure 3. It shows that the halo at the $4.6 \AA \mathrm{d}$-spacing hardly changes as the side-chain length increases. However, the intermolecular packing peak with the d-spacing of 14 to $19 \AA$ becomes sharper and more intense as the side-chain length increases. Moreover, there appears a new halo peak between these two halos, as clearly seen for poly-1, 2, 3, 4, as seen in Figure 3 and listed in Table III. This phenomenon is very similar to the WAXS results of poly(n-alkyl methacrylate) with alkyl groups greater than methyl, where the existence of local layer-like nano-scale order in amorphous state was proposed. . $^{17,18}$

Glass Transition Characteristics. The main chain of polynorbornene is considered to be very rigid with a quite
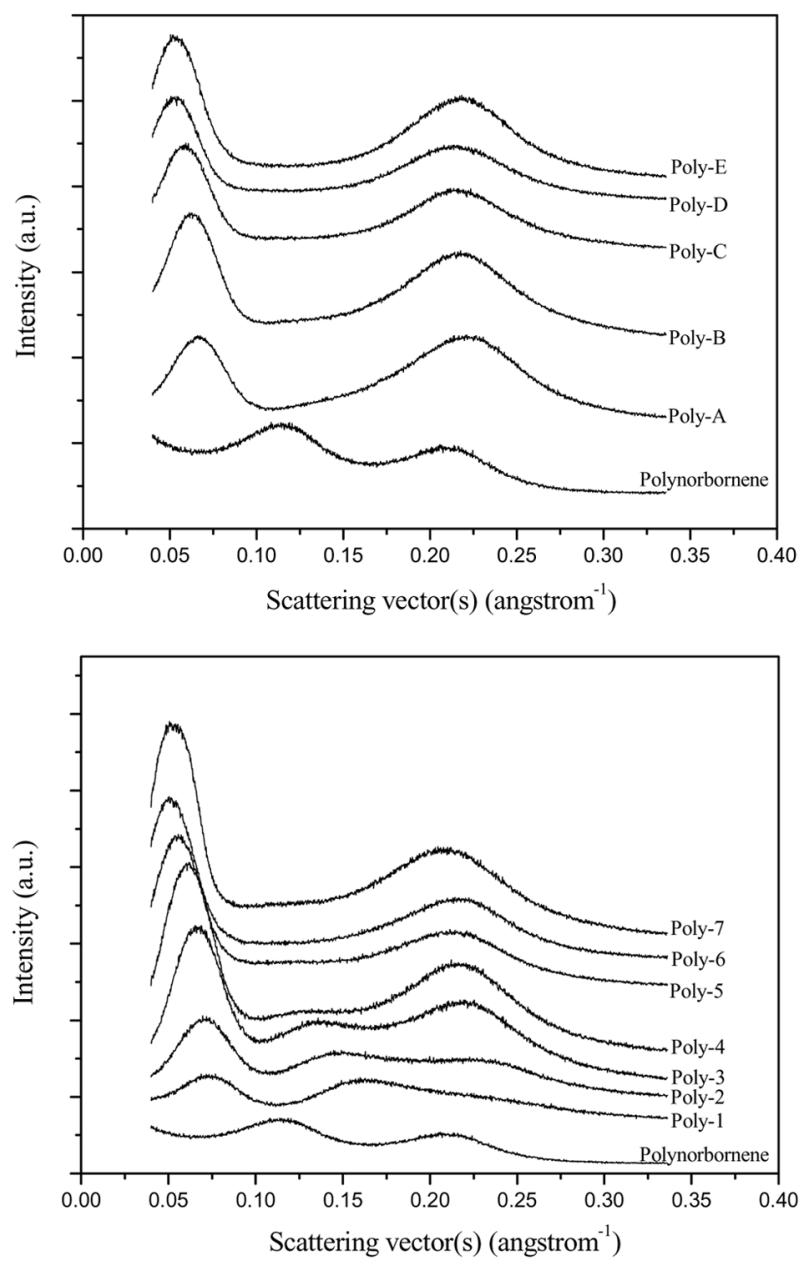

Figure 3. Wide-angle X-ray scattering patterns from powder samples of PNDMDs and PNDADAs with various alkyl side groups, plotted as function of the scattering vector, $s=2 \sin \theta_{B} /$ $\lambda$, where $2 \theta_{B}$ is the scattering angle and $\lambda$ is the wavelength of incident X-ray $(1.54 \AA)$.

Table III. Observed Bragg d-Spacings of WAXS Patterns

\begin{tabular}{cccc}
\hline & $\begin{array}{c}\text { First Peak } \\
(\AA)\end{array}$ & $\begin{array}{c}\text { Second Peak } \\
(\AA)\end{array}$ & $\begin{array}{c}\text { Third Peak } \\
(\AA)\end{array}$ \\
\hline Polynorbornene & 8.6 & & 4.8 \\
\hline Poly-A & 15.1 & - & 4.5 \\
Poly-B & 16.0 & - & 4.6 \\
Poly-C & 17.1 & - & 4.6 \\
Poly-D & 18.8 & & 4.6 \\
Poly-E & 18.7 & & 4.6 \\
\hline Poly-1 & 13.7 & 6.3 & 4.7 \\
Poly-2 & 14.2 & 6.8 & 4.5 \\
Poly-3 & 15.0 & 7.4 & 4.6 \\
Poly-4 & 16.3 & 7.5 & 4.6 \\
Poly-5 & 18.0 & - & 4.7 \\
Poly-6 & 19.5 & - & 4.5 \\
Poly-7 & 18.8 & - & 4.7 \\
\hline
\end{tabular}


high energy barrier for torsional rotations. Therefore, polynorbornene has a high glass transition temperature $T_{g}$, ca $370^{\circ} \mathrm{C}$, which nearly coincides with the onset of thermal decomposition. Therefore, vinyl-polynorbornene has a serious processing problem because the melt processing is not feasible and, moreover, its poor solubility prohibits solution processing.

The glass transition characteristics of polymers were measured by dynamic mechanical analyzer (DMA) in a dualcantilever mode with glass-fiber coated samples. Shear loss modulus $\left(G^{\prime \prime}\right)$ peaks were observed as the temperature was increased at a heating rate of $2 \mathrm{~K} / \mathrm{min}$ as shown in Figure 4, whereas no $T_{g}$ signal was seen in the differential scanning calorimetry (DSC) measurements. Therefore, the apparent $T_{g}$ was identified as the temperature of $G^{\prime \prime}$ maximum and shown in Table I and Figure 5.

PNDMD and PNDADA samples showed the same trend of decreasing $T_{g}$ as the alkyl side-chain length increases. However, at the same alkyl chain length, $T_{g}$ s of PNDMDs were found to be lower than those of PNDADAs. That is, the presence of the methylene group between norbornene and carboxylate moiety in poly-A, B, C, D, E series resulted in lower $T_{g} \mathrm{~s}$ than the corresponding $T_{g} \mathrm{~s}$ of poly-1,2,3,4,5.

As the alkyl chain length increases further, the $G^{\prime \prime}$ maximum was not clearly detected by DMA for poly-E and poly-7.

Dielectric and Optical Properties. Dielectric constant reflects the mobility of side groups in polynorbornene derivatives, since the polynorbornene backbone contains no permanent dipoles. Moreover, it is an important physical property of polymer insulators for information technology applications, since the signal propagation delay and power dissipation increase with increasing dielectric constant.

For PNDMDs (poly-A to E), the value of dielectric constant decreased from 3.5 to 3.0 as the alkyl chain length increased. Similarly, for PNDADAs (poly-1 to 6), the dielectric constant decreased from 3.3 to 2.6. At the same alkyl length, the dielectric constants of PNDMDs (poly-A to E) were found to be higher than those of PNDADAs (poly-1 to 5), as seen in Table I and Figure 5. This difference in the dielectric constants between PNDMDs and PNDADAs could arise from the variation in the packing density or the mobility of dipolar side groups. Therefore, in order to delineate the origin, separate measurements of refractive index were carried out. The measured values of refractive index, listed in Table I and plotted in Figure 5, do not show any significant differences between PNDMD and PNDADA samples with the same alkyl groups. Therefore, the higher values of the dielectric constants observed for PNDMD samples, as compared with those of PNDADAs with the same alkyl chains, could be attributed to the greater rotational mobility of the ester dipole groups of PNDMD in solid state.

It is also noted that the higher dipolar mobility of side groups observed for PNDMDs, as compared with the corresponding PNDADAs with the same alkyl chain length, coin-

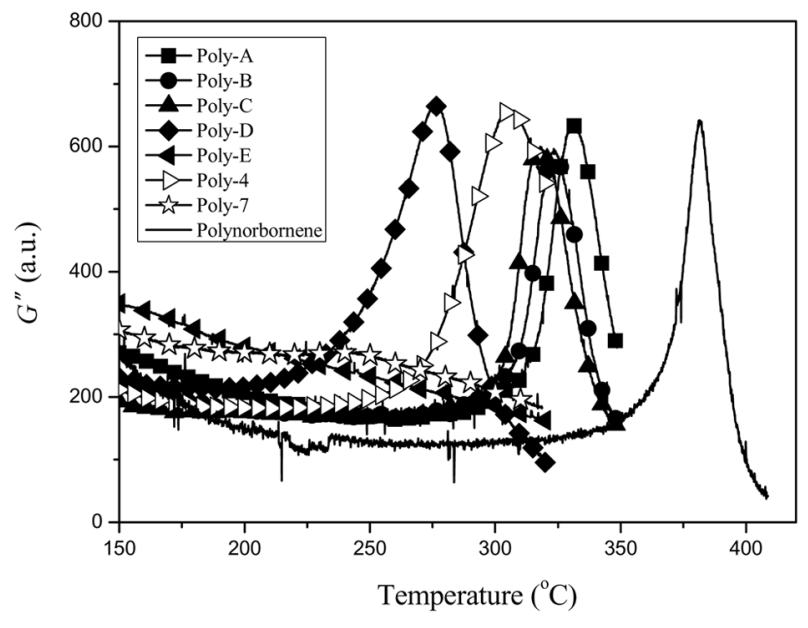

Figure 4. The shear loss modulus $G^{\prime \prime}$ plotted vs. temperature, obtained at a heating rate of $2 \mathrm{~K} / \mathrm{min}$.

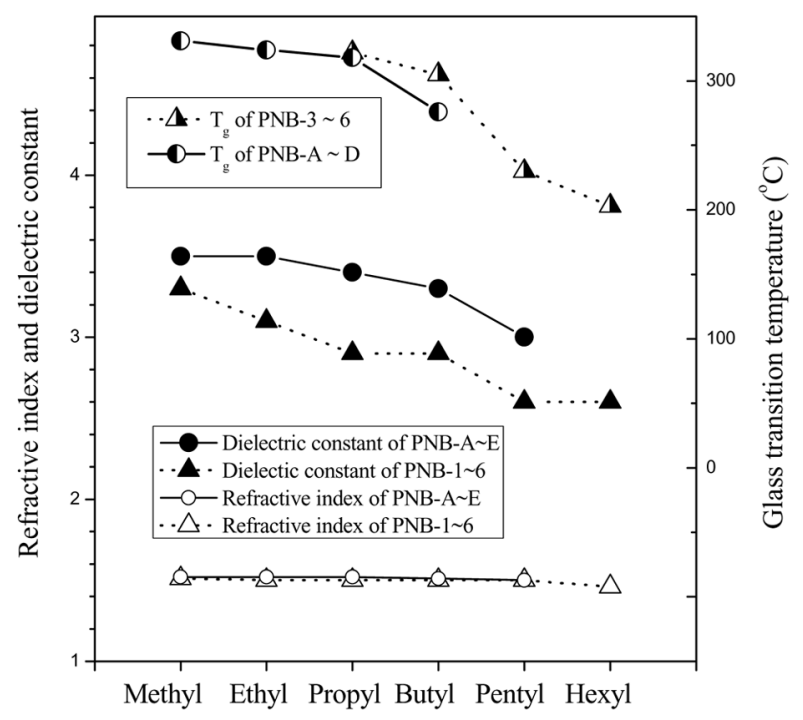

Figure 5. The variations of glass transition temperature, dielectric constant $(1 \mathrm{MHz})$, and refractive index $(632.8 \mathrm{~nm})$ with the alkyl substituents for both PNDMDs and PNDADAs.

cides with the lower $T_{g} \mathrm{~s}$ of PNDMDs than those of the corresponding PNDADAs in their glass transition characteristics. That is, the higher side-chain mobility of PNDMDs results in the lower glass transition temperature. In this regard, it appears that the local layer-like nano-scale order in amorphous PNDADAs, as seen by the WAXS results, is responsible for the reduced side-chain mobility.

\section{Conclusions}

Pure exo-norbornene dimethyl dicarboxylates with various alkyl substituents were successfully synthesized for high yield polymerization. Poly(norbornene dimethyl dicarboxylate)s, 
(PNDMD)s, were prepared by addition polymerization with palladium(II) catalyst from pure exo-monomers, and their structure and properties were compared with those of poly (norbornene dicarboxylic acid dialkyl ester)s, (PNDADA)s. Both polymer series exhibited good solubility in general organic solvents and excellent thermal stability up to $330^{\circ} \mathrm{C}$. WAXS studies indicated the presence of nano-scale layerlike order in amorphous PNDADAs, while PNDMD films showed random amorphous structure. The glass transition temperature and dielectric constant of polymers were found to decrease as the alkyl side-chain length increases. However, PNDMDs exhibited lower glass transition temperatures and higher dielectric constants, as compared with those of PNDADAs containing the same alkyl substituents, indicating that the higher side-group mobility results in the lower $T_{g}$. This difference in side-group mobility may be related to the nano-scale order in amorphous PNDADAs, which is likely to reduce the mobility of side groups.

Acknowledgements. We gratefully acknowledge the financial support by the Chemistry and Molecular Engineering Program of the Brain Korea 21 Project and Korean Ministry of Commerce, Industry and Energy. We also thank Prof. Ji Young Chang, Seoul National University and Prof. Andreas Greiner, Phillips-University of Marburg for helpful discussions and assistance with the polymer synthesis.

\section{References}

(1) C. Janiak and P. G. Lassahn, Macromol. Rapid Commun., 22, 479 (2001).

(2) B. Rieger, L. S. Baugh, S. Kacker, and S. Striegler, Late Transition Metal Polymerization Catalysis, $1^{\text {st }}$ Edition,
Wiley-VCH, Weinheim, 2003, Chapter 4.

(3) B. L. Goodall, G. M. Benedikt, L. H. McIntosh III, D. A. Barnes, and L. F. Rhodes, B.F. Goodrich Co., WO. 9514048 (1995).

(4) B. L. Goodall, W. Risse, and J. P. Mathew, B.F. Goodrich Co., WO. 9637526 (1996).

(5) N. R. Grove, P. A. Kohl, S. A. B. Allen, S. Jayaraman, and R. Shick, J. Polym. Sci.; Part B: Polym. Phys., 37, 3003 (1999).

(6) A. D. Hennis, J. D. Polley, G. S. Long, A. Sen, D. Yandulov, J. Lipian, G. M. Benedikt, L. F. Rhodes, and J. Huffman, Organometallics, 20, 2802 (2001).

(7) S. Breunig and W. Risse, Makromol. Chem., 193, 2915 (1992).

(8) J. K. Funk, C. E. Andes, and A. Sen, Organometallics, 23, 1680 (2004).

(9) B.-G. Shin, M.-S. Jang, D. Y. Yoon, and W. Heitz, Macromol. Rapid Commun., 25, 728 (2004).

(10) D. Craig, J. Am. Chem. Soc., 73, 4889 (1951).

(11) U. Okoroanyanwu, T. Shimokawa, J. Byers, and C. G. Willson, Chem. Mater., 10, 3319 (1998).

(12) B.-G. Shin, Ph.D. Thesis, Seoul National Univ., 2007. B. Liu, Y. Li, B.-G. Shin, D. Y. Yoon, I. L. Kim, L. Zhang, and W. Yan, in preparation.

(13) M. D. Wedlake and P. A. Kohl, J. Mater. Res., 17, 632 (2002).

(14) T. F. A. Hasselwander, W. Heitz, S. A. Krügel, and J. H. Wendorff, Macromol. Chem. Phys., 197, 3435 (1996).

(15) C. Zhao, M. R. Ribeiro, M. N. Pinho, V. S. Subrahmanyam, C. L. Gil, and A. P. Lima, Polymer, 42, 2455 (2001).

(16) W. J. Chung and P. J. Ludovice, ACS Polym. Mat. Sci. Eng., 89, 289 (2003).

(17) M. Beiner, Macromol. Rapid Commun., 22, 869 (2001). S. Hiller, O. Pascui, H. Budde, O. Kabisch, D. Reichert, and M. Beiner, New J. Phys., 6, 10 (2004).

(18) M. Wind, R. Graf, M. S. Renker, H. W. Spiess, and W. Steffen, J. Chem. Phys., 122, 014906 (2005). 